\title{
Habilidades Sociales y la Inteligencia Emocional de los estudiantes del II semestre estudios generales, Facultad de Educación. Universidad Nacional José Faustino Sánchez Carrión - Huacho 2018 \\ Social Skills and Emotional Intelligence of the students of the II semester general studies, Faculty of Education. José Faustino Sánchez Carrión National University - Huacho 2018
}

\author{
Elizabeth Julia Ramos Obregón ${ }^{1}$, Abelardo Rodolfo Campana Concha ${ }^{2}$
}

\begin{abstract}
RESUMEN
Objetivo: Identificar la relación entre las habilidades sociales y la inteligencia emocional de los estudiantes del II semestre, Facultad de Educación. Universidad Nacional José Faustino Sánchez Carrión - Huacho 2018. Materiales y Métodos: Es un estudio cuantitativo de diseño correlacional con una muestra de 206 estudiantes, a quienes se les aplicó dos cuestionarios. Se utilizó el programa SPSS, con un margen de error al 5\%. Resultados: La hipótesis general estableció una correlación de Rho = 0,838 que explica las habiliades sociales no se manifiestan regularmente lo mismo sucederá con la inteligencia emocional. Con respecto, a las cuatro hipótesis específicas se encontraron un nivel de relación de $\mathrm{Rho}=0,711, \mathrm{Rho}=0,664, \mathrm{Rho}=0,645$ y $\mathrm{Rho}=$ 0,441 , que en resumen dan a entender que irregular manifestación de las habilidades conductuales, cognitivas y fisiológicas se expresará de similar manera en la autoconciencia, autoregulación, motivación y empatía. Conclusión: Las habilidades referentes a la conducta, cognición y fisiología manifestados irregularmente se verán reflejados inadecuadamente en la inteligencia emocional.
\end{abstract}

Palabras clave: Habilidades sociales, inteligencia emocional, autoconciencia, autorregulaicón, motivación, empatía.

\begin{abstract}
Objective: To identify the relationship between social skills and emotional intelligence of the students of the second semester, Faculty of Education. José Faustino Sánchez Carrión National University - Huacho 2018. Materials and Methods: It is a quantitative study of correlational design with a sample of 206 students, to whom two questionnaires were applied. The SPSS program was used, with a margin of error of $5 \%$. Results: The general hypothesis established a correlation of Rho $=0.838$ that explains social skills do not manifest regularly the same will happen with emotional intelligence. Regarding the four specific hypotheses, a relationship level of Rho $=0.711$, Rho $=0.664$, Rho $=0.645$ and Rho $=0.441$ were found, which in summary suggest that irregular manifestation of behavioral, cognitive and physiological abilities will be expressed similarly in self-awareness, selfregulation, motivation and empathy. Conclusion: The skills related to behavior, cognition and physiology manifested irregularly will be inadequately reflected in emotional intelligence.
\end{abstract}

Keywords: Social skills, emotional intelligence, self-awareness, self-regulation, motivation, empathy.

\section{INTRODUCCIÓN}

Un problema común que nos permite alertar dificultades en las habilidades sociales es la manifestación de bullying en los centros educativos. Según la Encuesta Nacional de Relaciones Sociales realizada por el INEI (2015) alerta que alrededor del $75 \%$ de estudiantes ha sido víctima de agresiones por parte de sus compañeros alguna vez, asimismo consideran que el lugar más frecuente en el que ocurre la violencia es en el aula y durante clases. Por otro lado, según el portal Sí Se Ve Minedu (2018), el 20\% de los reportes de violencia escolar se deben al bullying en las escuelas. Estos problemas muchas veces no son afrontados a tiempo en las instituciones de educación básico, lo cual podría arrastrarse hasta su etapa adulta ya sea cuando inicia una vida universitaria o cuando se involucra en el campo laboral. Además el Minedu (2018) refiere: "El bullying es un fenómeno social cuya prevención requiere de una institución educativa donde se promueva el ejercicio y respeto de los derechos individuales y colectivos de los estudiantes, así como sus responsabilidades y la capacidad de reconocerse como sujetos capaces de actuar, participar y emitir una opinión, sin generar ni tolerar la discriminación".

Un informe publicado el 22 de junio por de la Agencia Andina (2019) en entrevista a especialistas refiere que una de las actividades del docente no solo es potenciar el aspecto académico, sino también las habilidades sociales de sus alumnos, el problema radica en que el educador se enfoca en capacitar con el aporte de conocimientos para la enseñanza académicas pero deja de lado este aspecto.

Reyes y Carrasco (2013) alerta que vivimos en una sociedad que se caracteriza por: "la continua globalización de los conocimientos en el terreno individual, estudiantil, profesional, laboral, la presión del reloj, la exigencia de un constante perfeccionamiento, entre otros factores, son situaciones que tienden a alterar el estado emocional de la mayoría de los estudiantes, llevándolos al borde de sus propios límites físicos y psíquicos".(p2)

En otras palabras, la complejidad del entorno que nos rodea implica que tengamos la capacidad de afrontar logros y dificultades, sin embargo no todos logran levantarse luego de una caída, puesto que no se cuenta con la inteligencia emocional adecuada, debido al poco o escaso énfasis que las instituciones educativas. Otro informe de la Agencia Andina (2019) publicado el 5 de julio, alerta la necesidad de potenciar la inteligencia emocional desde edades tempranas para que en su vida adulta puedan enfrentar frustraciones y sepan actuar frente a ello. Una alternativa que propone el especialista entrevista en este informe es el Coaching como alternativa para sacar el mayor potencial profesional para que la persona pueda dar lo mejor de sí, proporcionar su mejor versión a la vida y en el ámbito en 
el que se desenvuelve.

Este estudio pretende analizar la relación entre las Habilidades Sociales e Inteligencia Emocional de los estudiantes del IV semestre, Facultad de Educación UNMSM, 2018. En esta misma realidad se pretende conocer las relaciones entre habilidades sociales y la autoconciencia, autorregulación, motivación y empatía.

\section{MÉTODOS Y MATERIALES}

La investigación fue realizada con enfoque cuantitativo de nivel explicativo con un diseño correlacional.

La muestra estuvo conformada por 206 estudiantes. Para la obtención de los datos aplicó la técnica de encuesta a través del custionario. Para el tratamiento estadistico de las muestras se aplicó el programa APSS para la contrastación de las hipotesis.

\section{RESULTADOS}

\section{Prueba de hipótesis general}

HGA. Existe correlación significativa entre las habilidades sociales e Inteligencia emocional

Hg0. No existe correlación significativa entre las habilidades sociales e Inteligencia emocional

Tabla 1

Correlación de la hipótesis general

\begin{tabular}{lllc}
\hline \multicolumn{3}{c}{ Correlaciones } & \multicolumn{1}{c}{$\begin{array}{c}\text { Inteligencia } \\
\text { emocional }\end{array}$} \\
\hline & & $\begin{array}{l}\text { Coeficiente de } \\
\text { correlación }\end{array}$ & 0,838 \\
\cline { 3 - 3 } $\begin{array}{l}\text { Rho de } \\
\text { Sperman }\end{array}$ & $\begin{array}{l}\text { Habilidades } \\
\text { sociales }\end{array}$ & Sig. (bilateral) & 0,000 \\
\cline { 3 - 4 } & $\mathrm{N}$ & 121 \\
\hline
\end{tabular}

Con margen de error al $5 \%$, se halló una correlación muy buena de Rho $=0,838$ y el $p=0,000$. Resultado que expresa el rechazo de la hipótesis nula y la aceptación de la hipótesis alterna. Esto debido a que a la opinión mayoritaria de la muestra que refiere las habilidades sociales se relacionan a la inteligencia emocional aplicado a veces.

\section{Contrastación de la hipótesis específica 1}

H1. Existe correlación significativa entre las habilidades sociales y la autoconciencia.

Ho. No existe correlación significativa entre las habilidades sociales y la autoconciencia.

\section{Tabla 2}

Correlación hipótesis específica 1

\begin{tabular}{|c|c|c|c|}
\hline & Correlacione & & $\begin{array}{l}\text { Inteligencia } \\
\text { emocional }\end{array}$ \\
\hline \multirow{3}{*}{$\begin{array}{l}\text { Rho de } \\
\text { Sperman }\end{array}$} & \multirow{3}{*}{$\begin{array}{l}\text { Habilidades } \\
\text { sociales }\end{array}$} & $\begin{array}{l}\text { Coeficiente de } \\
\text { correlación }\end{array}$ & 0,711 \\
\hline & & Sig. (bilateral) & 0,000 \\
\hline & & $\mathrm{N}$ & 121 \\
\hline
\end{tabular}

Los resultados cuentan con margen de error al 5\%, del cual se evidenció una correlación buena de Rho $=0,711$ y el $p=0,000$, con ello se confirma el rechazo de la hipótesis nula y la aceptación de la hipótesis alterna, lo cual se debe a la opinión mayoritaria de la muestra, de quienes se alertó que las habilidades sociales se relacionan a la autoconciencia aplicado a veces.

\section{Contrastación de la hipótesis específica 2}

H2. Existe correlación significativa entre las habilidades sociales y la Autorregulación

Ho. No existe correlación significativa entre las habilidades sociales y la Autorregulación

Tabla 3

Correlación hipótesis especifica 2

\begin{tabular}{|c|c|c|c|}
\hline & \multicolumn{2}{|l|}{ Correlaciones } & Inteligencia \\
\hline \multirow{3}{*}{$\begin{array}{l}\text { Rho de } \\
\text { Sperman }\end{array}$} & \multirow{3}{*}{$\begin{array}{l}\text { Habilidades } \\
\text { sociales }\end{array}$} & $\begin{array}{l}\text { Coeficiente de } \\
\text { correlación }\end{array}$ & 0,664 \\
\hline & & Sig. (bilateral) & 0,000 \\
\hline & & $\mathrm{N}$ & 121 \\
\hline
\end{tabular}

Los resultados tienen un margen de error al $5 \%$, del cual se halló una correlación buena de Rho $=0,664$ y el $p=$ 0,000 , con ello se confirma el rechazo de la hipótesis nula y la aceptación de la hipótesis alterna, lo cual se debe a la opinión mayoritaria de la muestra, de quienes se alertó que las habilidades sociales se relacionan a la autorregulación aplicado a veces.

\section{Contrastación de la hipótesis específica 3}

H3. Existe correlación significativa entre las habilidades sociales y la motivación

Ho. No existe correlación significativa entre las habilidades sociales y la motivación

\section{Tabla 4}

Correlación hipótesis específica 3

\begin{tabular}{lllc}
\hline \multicolumn{3}{c}{ Correlaciones } & \multicolumn{1}{l}{$\begin{array}{l}\text { Inteligencia } \\
\text { emocional }\end{array}$} \\
\hline & & $\begin{array}{l}\text { Coeficiente de } \\
\text { correlación }\end{array}$ & 0,645 \\
\cline { 3 - 3 } $\begin{array}{l}\text { Rho de } \\
\text { Sperman }\end{array}$ & $\begin{array}{l}\text { Habilidades } \\
\text { sociales }\end{array}$ & Sig. (bilateral) & 0,000 \\
\cline { 3 - 4 } & $\mathrm{N}$ & 121 \\
\hline
\end{tabular}

Con un margen de error al $5 \%$, se estableció una correlación buena de Rho $=0,645$ y el $p=0,000$, con ello se ratifica el rechazo de la hipótesis nula y la aceptación de la hipótesis alterna, que en otras palabras se debe a la opinión mayoritaria de la muestra, de quienes se alertó que las habilidades sociales se relacionan a la motivación expresado casi siempre.

\section{Contrastación de la hipótesis específica 4}

H4. Existe correlación significativa entre las habilidades sociales y la Empatía

Ho. No existe correlación significativa entre las habilidades sociales y la Empatía 
Tabla 5

Correlación hipótesis específica 4

\begin{tabular}{llcc}
\hline & Correlaciones & & $\begin{array}{c}\text { Inteligencia } \\
\text { emocional }\end{array}$ \\
\hline & & $\begin{array}{l}\text { Coeficiente de } \\
\text { correlación }\end{array}$ & 0,441 \\
\cline { 3 - 4 } $\begin{array}{l}\text { Rho de } \\
\text { Sperman }\end{array}$ & $\begin{array}{l}\text { Habilidades } \\
\text { sociales }\end{array}$ & Sig. (bilateral) & 0,000 \\
\cline { 3 - 4 } & & $\mathrm{N}$ & 121 \\
\hline
\end{tabular}

\section{DISCUSIÓN}

En la hipótesis general se encontró una correlación muy buena de Rho = 0,838 entre las variables expuestas en este estudio. En la variable habilidades sociales la mayoría representado por el $55,4 \%$ considera que a veces sabe manejar sus habilidades sociales, un $43,0 \%$ casi siempre. Por otro lado, la inteligencia emocional, es a veces aplicado por el 52,9\%, mientras que el $44,6 \%$ casi siempre. Con ello evidenciamos que los manejos inadecuados de las habilidades sociales se relacionan al manejo de sus emociones en su proceso formativo universitario.

En el planteamiento de la hipótesis específica 1 , se confirmó la existencia de una correlación buena de Rho $=0,711$, esto se explica en la opinión mayoritaria de la muestra, al señalar que el $55,4 \%$ a veces sabe manejar sus habilidades sociales, seguido del $43,0 \%$ que casi siempre sabe manejarlo; del mismo modo, la dimensión autoconciencia es a veces aplicado por el 52,1\%, seguido de un $41,3 \%$ como casi siempre. En resumen, el desconocimiento de no saber manejar idóneamente las habilidades sociales se relaciona a la irregular frecuencia en la que puede reconocer sus fortalezas, corregir sus debilidades e incluso retroalimentar sus conocimientos.

En el planteamiento de la hipótesis específica 2, se halló una correlación buena de Rho $=0,664$, esto fue evidenciado en la opinión mayoritaria de los estudiantes, al señalar que el $55,4 \%$ a veces sabe manejar sus habilidades sociales, seguido del $43,0 \%$ que casi siempre sabe manejarlo; de igual manera, en la autorregulación el $48,8 \%$ opina a veces lo aplican, mientras que el $43,0 \%$ casi siempre. Con lo cual, se entiende el desconocimiento en cuanto al adecuado proceder de las habilidades sociales se relaciona a la irregular frecuencia en la que pueden dominar sus impulsos, pensar con claridad, análisis de sus aprendizajes, su accionar ético, incluso en su planteamiento metodológico e innovador para estudiar.

En el planteamiento de la hipótesis específica 3, encontró una correlación buena de Rho $=0,645$, lo cual es el resultado de la opinión mayoritaria de la muestra, cuyo $55,4 \%$ afirman que a veces sabe aplicar sus habilidades sociales, mientras que el $43,0 \%$ indica que casi siempre. Por otro lado, el $45,5 \%$ actúa con motivación, mientras que el $35,5 \%$ solo a veces. Con se observa que el inadecuado proceder en las habilidades sociales se relaciona con en la irregular frecuencia en la que se esfuerza para alcanzar sus metas, en las decisiones para el planteamiento de mejores cosas para sus desarrollo personal y profesional.

En el planteamiento de la hipótesis específica 4 , determinó una correlación moderada de Rho $=0,441$ cuyos resultados se deben a las afirmaciones entre las habilidades sociales y la empatía. La mayoría representado por el $55,4 \%$ indica a veces es capaz a veces cuenta con las habilidades sociales necesarias, seguido del $43,0 \%$ considera que casi siempre; por otro lado, el $47,9 \%$ a veces actúa con empatía, mientras que el $43,8 \%$ casi siempre lo es. Por tanto, el desconocimiento de no saber manejar idóneamente las habilidades sociales se relaciona a la irregular frecuencia en la que actúan con empatía con sus compañeros en cuanto a la necesidad de aprendizaje, sus retos de estudio, sus éxitos o sus orígenes.

Por consiguiente, este estudio permitió demostrar que los dificultades en las habilidades sociales de los estudiantes está relación a la inteligencia emocional de los estudiantes, cuya opinión es compartida también con los de Verde (2015) consideró prudente que los docentes brinden a alumnos las herramientas necesarias que les permitan afrontar los conflictos y agresiones desde el momento en que surgen, creando un buen ambiente en el aula que favorezca la convivencia, debido a que el error que suelen cometer los docentes es que asumen que todos los estudiantes saben manejar sus habilidades sociales, cosa que no es así puesto que hemos identificado que solo a veces son capaces de expresar dichas habilidades.

Por otro lado, los estudios de Sastre (2017) han demostrado que los niveles adecuados de la inteligencia emocional ayudan a afrontar con mayor éxito a los contratiempos cotidianos y el estrés laboral al que se enfrentan los profesores de secundaria en su centro de trabajo, esto no es una realidad ajena al docente y estudiante.

Con respecto al análisis de la hipótesis general confirmó que las habilidades sociales considerado de a veces aplicado, en especial de los aspectos fisiológicos; se relaciona a la inteligencia emocional, manifestado por los estudiantes con una regularidad de a veces. Concluyendo que las habilidades referentes a la conducta, cognición y fisiología manifestados irregularmente se verán reflejados inadecuadamente en la inteligencia emocional.

La hipótesis específica 1 comprobó que las habilidades sociales considerado de a veces, incluso en los aspectos conductuales; se relaciona a la autoconciencia, manifestado por la muestra con una regularidad de a veces. En consecuencia, la irregularidad en la que los estudiantes expresan las habilidades sociales disminuye la posibilidad de que la capacidad de reconocer sus fortalezas, corregir sus debilidades e incluso retroalimentar sus conocimientos.

La hipótesis específica 2 halló una relación entre las habilidades sociales considerado de a veces, incluso en los aspectos cognitivos; y la autorregulación manifestado por la muestra con una regularidad de a veces. En síntesis, la irregularidad en la que los estudiantes expresan las habilidades sociales impide que puedan adecuar el dominio de sus impulsos, la claridad de su pensamiento, su accionar ético y el planteamiento metodológico e innovador para estudiar.

La hipótesis específica 3 encontró una relación entre las habilidades sociales considerado de a veces, incluso en los aspectos fisiológicos; y la motivación que es expresado por la muestra casi siempre. En conclusión, si las habilidades sociales no se manifiestan de forma constante se harán evidente en el interés de los estudiantes en cuanto al alcance sus metas, sus decisiones para el planteamiento de mejores cosas para sus desarrollo personal y profesional. 
La hipótesis específica 4 identificó que las habilidades sociales considerado de a veces aplicado, incluso en los conductual, cognitivo y fisiológico; se relaciona a la empatía, manifestado por los estudiantes con una regularidad de a veces. Demostrando que la irregularidad en la que los estudiantes expresan las habilidades sociales impide que puedan adecuar empáticamente con sus compañeros en cuanto a la necesidad de aprendizaje, sus retos de estudio, sus éxitos o sus orígenes.

Asi mismo, este estudio permitió demostrar que los dificultades en las habilidades sociales de los estudiantes está relación a la inteligencia emocional de los estudiantes, cuya opinión es compartida también con los de Verde (2015) consideró prudente que los docentes brinden a alumnos las herramientas necesarias que les permitan afrontar los conflictos y agresiones desde el momento en que surgen, creando un buen ambiente en el aula que favorezca la convivencia, debido a que el error que suelen cometer los docentes es que asumen que todos los estudiantes saben manejar sus habilidades sociales, cosa que no es así puesto que hemos identificado que solo a veces son capaces de expresar dichas habilidades.

Por otro lado, los estudios de Sastre (2017) ha demostrado que los niveles adecuados de la inteligencia emocional ayudan a afrontar con mayor éxito a los contratiempos cotidianos y el estrés laboral al que se enfrentan los profesores de secundaria en su centro de trabajo, esto no es una realidad ajena al docente y estudiante.

\section{CONCLUSIONES}

El análisis de la hipótesis general confirmó que las habilidades sociales considerado de a veces aplicado, se relaciona a la inteligencia emocional, manifestado por los estudiantes con una regularidad de a veces. Por tanto las habilidades referentes a la conducta, cognición y fisiología manifestados irregularmente se verán reflejados inadecuadamente en la inteligencia emocional.

\section{REFERENCIAS BIBLIOGRÁFICAS}

INEI (2015). Encuesta Nacional de Relaciones Sociales. Lima: Instituto Nacional de Estadística e Informática. $\mathrm{R}$ e c u p e r a d o e $n$ https://webinei.inei.gob.pe/anda inei/index.php/ddi browser/581/export/?format=pdf\&generate=yes

Minedu (2018). Guía para aprender y atender el acoso a e s t ud i a n t e s. Re cu perado e n http://www.siseve.pe/Web/file/materiales/Prevenci \%C3\%B3n-Atenci\%C3\%B3n.pdf

Portal de Agencia Andina.(5 julio 2019) Coaching: colegios deben potenciar inteligencia emocional en menores. Recuperado en ( 5 de julio de 2019)https://andina.pe/agencia/noticia-coachingcolegios-deben-potenciar-inteligencia-emocionalmenores-757684.aspxPortal de Agencia Andina. Profesores deben potenciar tanto habilidades sociales como académicas. Recuperado en (22 de junio de 2019) https://andina.pe/agencia/noticiaprofesores-deben-potenciar-tanto-habilidadessociales-como-academicas-511326.aspx

Portal de Agencia Andina (22 junio 2019) Profesores deben potenciar tanto habilidades sociales como académicas. (22 de junio de 2019). Agencia Andina. Recuperado en https://andina.pe/agencia/noticiaprofesores-deben-potenciar-tanto-habilidadessociales-como-academicas-511326.aspx

Reyes, C. y Carrasco, I. (2013). Inteligencia emocional en estudiantes de la Universidad Nacional del Centro. Apuntes de Ciencias Sociales, 4 (1), p.87 100.

Sastre, P. (2017). El desgaste profesional y la inteligencia emocional en la acción profesional (Tesis Posgrado) Universidad de Granada, España. Recuperado en: https://hera.ugr.es/tesisugr/26770830.pdf

Verde, R. (2015). Taller aprendiendo a convivir para el desarrollo de habilidades sociales en los alumnos del primer año de educación secundaria de la I.E. Víctor Raúl Haya de la Torre El Porvenir - Trujillo, 2014 (Tesis Posgrado) Universidad Privada Antenor Orrego, Trujillo, Perú. 